\title{
Performance characteristics of a gas proportional scintillation counter coupled to a microstrip gas chamber photosensor
}

\author{
J.F.C.A. Veloso*, J.M.F. dos Santos, C.A.N. Conde \\ Departamento de Física, Universidade de Coimbra, P 3000 Coimbra, Portugal
}

\begin{abstract}
A P10-filled microstrip gas chamber (MSGC) is used to replace the conventional photomultiplier tube (PMT) as the photosensor for a gas proportional scintillation counter (GPSC). The vacuum ultra-violet (VUV) scintillation light produced in the xenon-filled GPSC is transmitted through a $1 \mathrm{~mm}$ thick high-purity quartz window to the MSGC where it is converted to photoelectrons by a CsI photocathode deposited directly onto the surface of a microstrip plate (MSP). These photoelectrons are afterwards multiplied near the microstrip plate anodes with a charge gain of about $10^{3}$. The energy resolution achieved for $5.9 \mathrm{keV} \mathrm{X-rays} \mathrm{is} 11.5 \%$ which, while not yet as good as the $8 \%$ figure for standard GPSC (instrumented with a PMT), is already better than the energy resolution obtained for standard proportional counters. Experimental results are presented and discussed. With this design a compact GPSC is obtained which has the further advantage of being much less sensitive to magnetic fields than PMT-based GPSCs. (C) 1999 Elsevier Science B.V. All rights reserved.
\end{abstract}

Keywords: Gas proportional scintillation counter; Microstrip gas chamber photosensor

\section{Introduction}

Gas proportional scintillation counters (GPSC) are used to detect $\mathrm{X}$-rays over a range of energies from about $200 \mathrm{eV}$ up to $100 \mathrm{keV}$ [1-4]. These detectors produce a large secondary scintillation which leads to large amplitude pulses with low statistical fluctuations and, therefore, they provide well-documented advantages over conventional gas proportional counters. GPSCs are usually filled with noble gases, typically xenon, and combine large detection areas with good energy resolution

\footnotetext{
* Corresponding author.
}

and almost linear energy response even at elevated counting rates.

A typical gas proportional scintillation counter uses a quartz window photomultiplier tube (PMT) to detect the secondary VUV scintillation from its noble gas filling [1-4]. These PMTs are expensive, fragile, power consuming and bulky and do not lend themselves to compact designs. Attempts to replace the PMT in a GPSC with a more convenient photosensor have included a multiwire proportional chamber (MWPC) with a photosensitive TMAE gas filling [5] or with a CsI photocathode inside the chamber $[6,7]$.

Following the introduction of the microstrip gas chamber (MSGC) by Oed in 1988 [8], several 
authors have demonstrated the feasibility of detecting VUV light with a CsI photocathode layer deposited directly onto a microstrip plate $[9,10]$. In its basic form, the microstrip plate (MSP) consists of thin metal electrode strips photolithographed onto an insulating substrate with a very small spacing between parallel alternating anodes and cathodes with a pitch of a few hundred micrometers. A low voltage applied between the closely spaced electrodes produces the intense electric field required for charge multiplication in the gas medium while facilitating the fast removal of residual positive ions. This design results in a very compact detector with reduced space-charge effects, high rate capabilities and stable gains in the $10^{3}-10^{4}$ range, in addition to inherent spatial sensitivity. MSGCs have found success in diverse fields such as high-energy physics, X-ray astronomy, medicine and EDXRS applications [11-15].

Since microstrip detectors are simpler, more rugged, compact and inexpensive than the attempted solutions based on MWPC mentioned above, we have attempted to develop a MSGC-based photosensor for a GPSC. First results have been obtained with a microstrip plate (within the xenon envelope of the GPSC) with a CsI photocathode deposited directly onto its surface [16]. The performance of this system has been marginal $(17 \%$ and $6 \%$ energy resolution for 5.9 and $60 \mathrm{keV} \mathrm{X-rays,} \mathrm{respectively,}$ with a large tail due to noise extending up to $1.4 \mathrm{keV})$. Efforts to understand the performance limitations have been discussed [16] and attributed to optical positive feedback, since further VUV scintillation is produced in avalanche processes that take place near the MSP anodes in xenon atmosphere. As a result, the MSGC gain and, hence, the overall detector performance is limited.

To overcome this positive feedback limitations, we have developed a system with a thin quartz window separating the xenon GPSC from a microstrip gas-photosensor chamber, now filled with a nonscintillating gas. The filling gas for the MSGC can then be selected (other than pure xenon) amongst noble gas mixtures with quenching agents to reduce feedback and allow higher gas gains. Preliminary results showing an energy resolution similar to the one for previous design [16] and not better than that of standard proportional counters were presented at a conference [17]. However, in the present work we describe the details of an improved system which presents an energy resolution already superior to that of standard proportional counters. Experimental results for the energy resolution, gain and operational stability for different GPSC and MSGC voltages are presented.

\section{Description}

The new design of a xenon GPSC having an MSGC as photosensor is depicted in Fig. 1. Primary electrons produced in the GPSC absorption region by $\mathrm{X}$-ray drift towards the scintillation region under the influence of a low-intensity electric

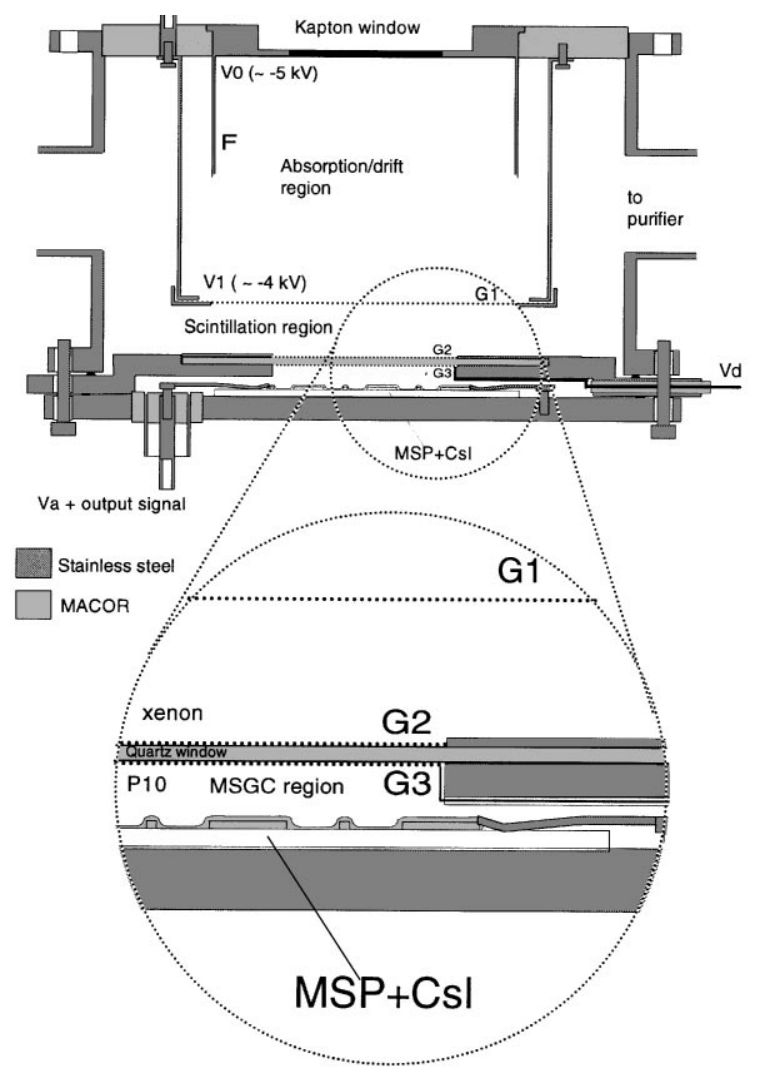

Fig. 1. Experimental system design comprising the GPSC and the MSGC coupled with a quartz window. 
field. In the scintillation region, under a stronger electric field (below ionisation threshold), electron-xenon collisions excite xenon atoms which produce excimers that decay emitting scintillation photons in the VUV $(7.2 \mathrm{eV}$, i.e. $170 \mathrm{~nm})$. A fraction of the VUV photons produced are transmitted through the quartz window to the MSG chamber and reach the CsI layer deposited onto the MSP surface. VUV photons release photoelectrons from the CsI (photocathode). These photoelectrons initiate charge avalanches in the strong electric field region around the anodes of the microstrip plate.

The GPSC gas volume is separated from the MSGC volume by a $1 \mathrm{~mm}$ thick high-purity quartz (Spectrosil) window. The GPSC is filled with pure xenon which circulates by convection through a SAES ST707-based purifier. The absorption/drift region is located between the radiation entrance window and the first grid, G1, which is made of stainless steel and has an optical transparency of about $95 \%$. A focusing electrode, F, at the same potential as the entrance window, concentrates the primary electron cloud closer to the axis. The electron collection grid, G2, is a high-transparency chromium grid evaporated onto the quartz window, in its GPSC side.

Another high-transparency chromium grid G3 is deposited onto the quartz window, in its MSGC side and lined up with G2 lines to reduce optical absorption. G3 is used to polarise the $4 \mathrm{~mm}$ drift region of the MSGC. This chamber is filled with a P10 mixture at about atmospheric pressure and the gas flows continuously. The photocathode consists of a $500 \mathrm{~nm}$ CsI layer, $30 \mathrm{~mm}$ in diameter, vacuum deposited onto the surface of the MSP (a CERN MS-4 design with a $30 \times 30 \mathrm{~mm}^{2}$ effective area, produced by IMT-Masken und Teilungen, Switzerland). It consists of $10 \mu \mathrm{m}$ anodes and $80 \mu \mathrm{m}$ cathodes with a $200 \mu \mathrm{m}$ pitch. Both are made of $0.2 \mu \mathrm{m}$ thick chromium film deposited onto a $300 \mu \mathrm{m}$ Desag D263 glass substrate. The backplane is a flat, nonstructured, $0.1 \mu \mathrm{m}$ thick chromium layer. All the anodes are connected together as well as the cathodes.

The transparency of the P10 mixture to $170 \mathrm{~nm}$ UV photons is close to $100 \%$ [9] and that of the $1 \mathrm{~mm}$ thick quartz window is better than $90 \%$ [18]. Thus, UV photons travel from the scintillation region to the CsI photocathode with little absorption.

Since the photoelectrons are ejected from the photocathode deposited onto the MSP, and then drift a very short distance (about $50 \mu \mathrm{m}$ ) toward the anodes, the MSGC photosensor is much more immune to magnetic fields than a PMT.

The absorption efficiency of the MSGC for Xrays can be neglected when compared to that of the xenon-filled GPSC.

Anode pulses are preamplified with a CANBERRA 2006 charge-to-voltage preamplifier unit and afterwards linearly amplified, and pulse height analysed. Amplifier time constants of $5 \mu$ s for the ${ }^{55} \mathrm{Fe} \mathrm{X}$-ray source pulses were chosen for the results reported here. For pulse amplitude and energy resolution measurements the $5.9 \mathrm{keV} \mathrm{X}$-ray pulseheight distributions are fitted to a Gaussian function superimposed on a linear background.

\section{Experimental results and analysis}

In Fig. 2 we present the pulse amplitude as a function of the reduced electric field, $E / p$, in the scintillation region, while maintaining constant the MSGC gain and the reduced electric field in the absorption region. The voltage between the anodes and cathodes of the MSGC photosensor, $V_{\mathrm{a}}$, was

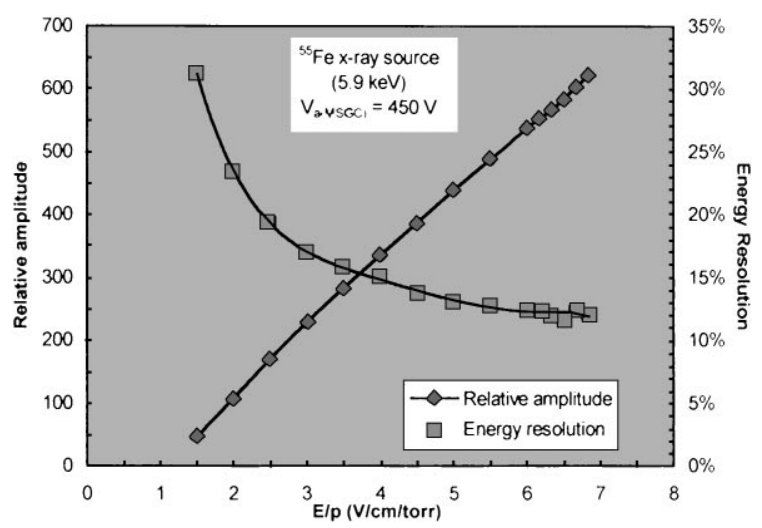

Fig. 2. Variation of the pulse amplitude and energy resolution with the reduced electric field intensity, $E / p$, in the scintillation region for $5.9 \mathrm{keV}$ X-rays for the xenon-filled GPSC coupled to a P10-filled MSGC photosensor. 
maintained at $450 \mathrm{~V}$. As expected, the measured pulse amplitude presents the linear dependence characteristics of the secondary scintillation intensity on the reduced electric field, with a threshold at about $1 \mathrm{~V} \mathrm{~cm}^{-1}$ Torr $^{-1}$. As shown, the detector energy resolution improves with increasing $E / p$.

For performance comparison, the above study was also performed for the GPSC instrumented with a PMT instead of the MSGC photosensor. The PMT was placed below and close to the quartz window to collect the scintillation light. The photomultiplier tube used was an EMI 9266MGF2B with eight dynodes and $\mathrm{MgF}_{2}$ window which has a high transparency at $170 \mathrm{~nm}$. The obtained experimental results for the PMT together with the ones obtained with the MSGC photosensor are presented in Fig. 3. For high $E / p$, the energy resolution of the GPSC coupled to the MSGC photosensor approaches the energy resolution of the GPSC coupled to the PMT photosensor. This resolution is already better than the one for standard proportional counters.

A typical pulse-height distribution for a $5.9 \mathrm{keV}$ $\mathrm{K}_{\alpha}$ line of the ${ }^{55} \mathrm{Fe} \mathrm{X}$-ray source is depicted in Fig. 4, presenting a low background down to about $500 \mathrm{eV}$.

The GPSC/MSGC pulse amplitude and energy resolution for $5.9 \mathrm{keV} \mathrm{X}$-rays as a function of anode

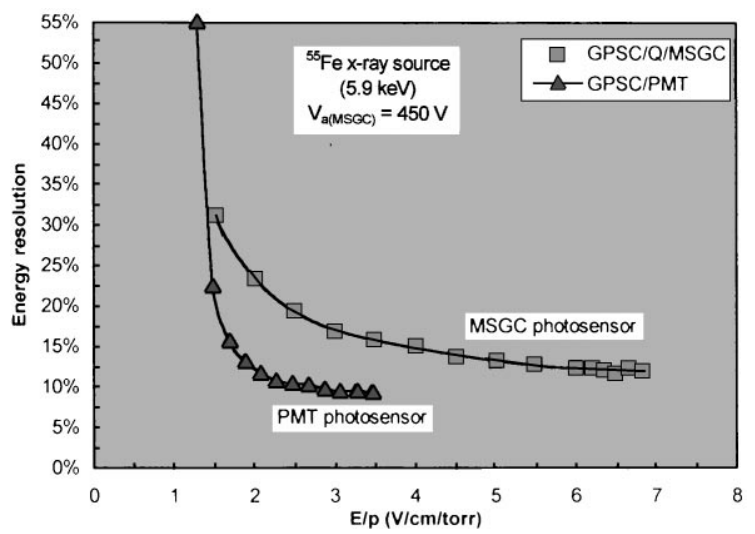

Fig. 3. Energy resolution for $5.9 \mathrm{keV} \mathrm{X}$-rays as a function of the reduced electric field in the scintillation region for the GPSC coupled to a P10-filled MSGC photosensor (GPSC/Q/MSGC) and for the GPSC coupled to a PMT photosensor (GPSC/PMT).

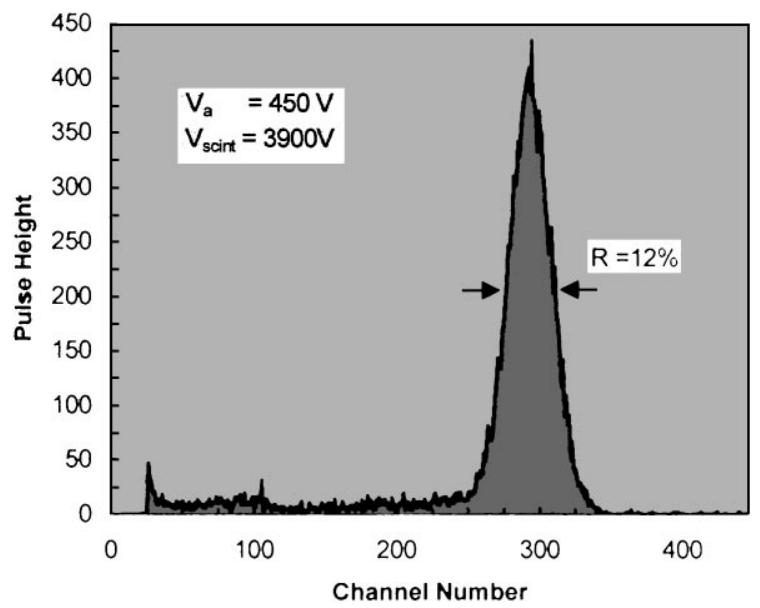

Fig. 4. Typical pulse-height distributions for $5.9 \mathrm{keV}$ X-rays of the gas proportional scintillation counter coupled to a P10-filled MSGC photosensor.

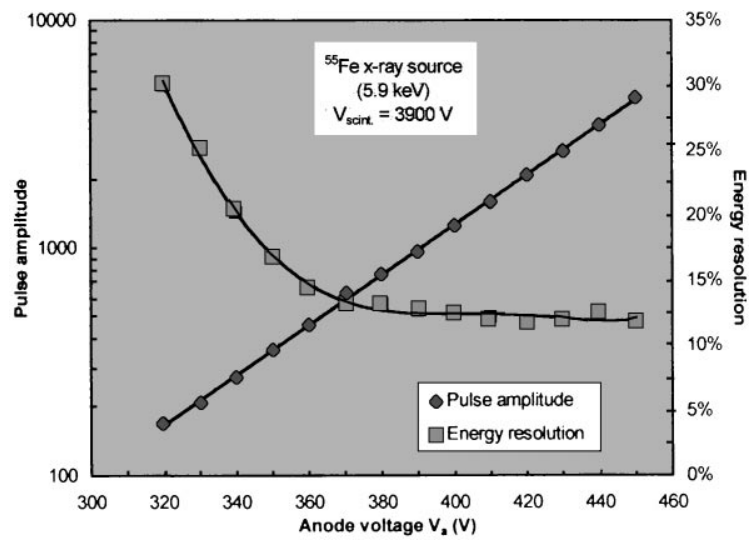

Fig. 5. Variation of the pulse amplitude and energy resolution with the MSGC photosensor anode voltage, $V_{\mathrm{a}}$, for a constant reduced electric field intensity in the xenon GPSC scintillation region, and for $5.9 \mathrm{keV} \mathrm{X}$-rays.

to cathode MSGC voltage, $V_{\mathrm{a}}$, is presented in Fig. 5. The reduced electric field in the scintillation region and the drift field of the GPSC were maintained at 6 and $0.3 \mathrm{~V} \mathrm{~cm}^{-1} \mathrm{Torr}^{-1}$, respectively. As expected the pulse amplitude increases exponentially and the energy resolution improves with increasing $V_{\mathrm{a}}$. The best energy resolutions are obtained for $V_{\mathrm{a}}$ voltages above $400 \mathrm{~V}$, until electrical breakdown takes place at about $460 \mathrm{~V}$. 
The detector energy resolution was found to be independent of the MSGC gas pressure in the 700-1000 Torr range.

Preliminary results of the short-term temporal stability ( $2 \mathrm{~h}$ ) of the GPSC/MSGC detector show relative pulse-amplitude variations below $2 \%$.

\section{Conclusions}

We have developed a xenon-filled gas proportional scintillation counter coupled to a P10-filled microstrip gas chamber photosenor. The best energy resolution achieved for $5.9 \mathrm{keV} \mathrm{X}$-rays is $11.5 \%$ which, while not yet as good as the $8 \%$ figure for standard GPSC (instrumented with a PMT), is already better than the energy resolution obtained for standard proportional counters. This new type of GPSC has the advantage of being capable of large window areas together with relative insensitivity to magnetic fields.

\section{Acknowledgements}

This work was carried out in the Atomic and Nuclear Instrumentation Group of the Instrumentation Centre (Unit 217/94) of the Physics Department, University of Coimbra. Support is acknowledged from Fundação para a Ciência e Tecnologia (FCT), through project CERN/P/FAE/1163/97. J.F.C.A. Veloso was supported by a research grant from FCT (PRAXIS XXI/BD/11231/97) and acknowledges travel support by Fundação Luso Americana para o Desenvolvimento (FLAD).

\section{References}

[1] J.M.F. dos Santos, A.C.S.S. Bento, C.A.N. Conde, X-ray Spectrometry 22 (1993) 328.

[2] A. Peacock, R.D. Andresen, E.A. Leimman, G. Manzo, B.G. Taylor, Nucl. Instr. and Meth. 169 (1980) 613.

[3] A. Smith, M. Bavdaz, Rev. Sci. Instr. 63 (1992) 689.

[4] J.F.C.A. Veloso, J.M.F. dos Santos, C.A.N. Conde, IEEE Trans. Nucl. Sci. NS- 42 (1995) 369.

[5] D.F. Anderson, Nucl. Instr. and Meth. 178 (1980) 125.

[6] V. Dangendorf, A. Breskin, R. Chechik, H. Schmidt-Böcking, Nucl. Instr. and Meth. A 289 (1990) 322.

[7] J.A.M. Lopes, J.M.F. dos Santos, C.A.N. Conde, R.E. Morgado, IEEE Trans. Nucl. Sci. NS- 44 (1997) 517.

[8] A. Oed, Nucl. Instr. and Meth. A 263 (1988) 351.

[9] K. Zeitelhack, J. Friese, R. Gernhäuser, P. Kienle, H.-J. Körner, P. Maier-Komor, S. Winkler, Nucl. Instr. and Meth. A 351 (1994) 585.

[10] F. Angelini, R. Belazini, M. Bozzo, A. Braem, A. Brez, V. Lebedenko, M.M. Massai, R. Raffo, G. Spandre, M.A. Spezziga, Nucl. Instr. and Meth. A 371 (1996) 358.

[11] M. Bedjidian et al., Nucl. Phys. B 32 (1993) 189.

[12] F. Angelini, R. Belazini, L. Bosisio, A. Brez, M.M. Massai, G. Spandre, M.R. Torquati, Nucl. Instr. and Meth. A 323 (1992) 229.

[13] C. Budtz-Jorgensen, A. Bahnsen, C. Olesen, M.M. Madsen, P. Jonasson, H.W. Schnopper, A. Oed, Nucl. Instr. and Meth. A 310 (1991) 82.

[14] Sauli Fabio, Nucl. Instr. and Meth. A 323 (1992) 1.

[15] J.F.C.A. Veloso, J.M.F. dos Santos, C.A.N. Conde, R.E. Morgado, X-ray Spectrometry 26 (1997) 237.

[16] J.F.C.A. Veloso, J.A.M. Lopes, J.M.F. dos Santos, C.A.N. Conde, IEEE Trans. Nucl. Sci. NS- 43 (4) (1996) 1232.

[17] J.F.C.A. Veloso, J.M.F. dos Santos, C.A.N. Conde, Conf. Record of the 1996 IEEE Nuclear Science Symp., vol. I, 1997, pp. 73-76.

[18] THORN EMI Electron Tubes Catalogue, 1993. 